\title{
High Fidelity Simulation and the Development of Clinical Judgment in Senior Nursing Students: A Mixed Method Approach
}

\author{
Warongrong Nilphet ${ }^{1}$ \\ ${ }^{1}$ Pediatric Nursing Department, Boromarajonani College of Nursing Songkhla, Songkhla, Thailand \\ Correspondence: Warongrong Nilphet, Faculty of Pediatric Nursing Department, Boromarajonani College of \\ Nursing Songkhla, Songkhla, Thailand. Tel: 66-74-311-890.
}

Received: April 14, 2021 Accepted: August 21, 2021 Online Published: August 27, 2021

doi:10.5539/gjhs.v13n10p19

URL: https://doi.org/10.5539/gjhs.v13n10p19

\begin{abstract}
Clinical judgment is defined as an understanding and interpretation regarding patient's needs, health problems or concerns (Tanner, 2006). There are four interrelated processes in Tanner's model that consist of noticing, interpreting, responding, and reflecting (Tanner, 2006). Because clinical judgment is extremely complex and encompasses many ways of thinking and types of knowledge, it requires a flexible capability to identify significant features of indeterminate clinical circumstances. Mixed methods study was conducted to describe senior nursing students' experience in using high-fidelity simulation to evaluate the development of clinical judgment skills. A convenience sampling of 30 senior nursing students who signed the consent, met the inclusion criteria, and attend the selected school of nursing in the fall of 2020 were used for this study. All participants answered questionnaires regarding the quantitative survey. Participants interviewed face-to-face and video call using Zoom meeting program and recorded using an audio recorder. Both the quantitative and qualitative findings identified that learning through high-fidelity simulation supports the improvement in the participants' clinical judgment skills. All participants reported their perceptions and experiences from using high-fidelity simulation develop and support their clinical judgment skills from the beginning through the end of the simulation, especially improving prioritizing data and working as a team with providing effective communication.
\end{abstract}

Keywords: high-fidelity simulation, clinical judgment, senior nursing student

\section{Introduction}

\subsection{Introduce the Problem}

NSI Nursing Solution (2021) reported that the turnover of registered nurses increased by 2.8 percent in 2020 and currently stands at $18.7 \%$. The average hospital turnover for registered nurses rate is up to over 83 percent of the workforce since 2016. At present, lack of nursing experience has been identified as a major predictor of nurses' turnover and is exacerbated by factors such as compassion fatigue in nurses working in clinical settings (ANA, 2016). In particular, some specialized clinical settings such as the Intensive Care Unit (ICU), Progressive Care Unit (PCU), Cardiac Care Unit (CCU), Pediatric Intensive Care Unit (PICU), or Newborn Intensive Care Unit (NICU) have been found to be the most challenging areas for newly graduated nurses (Jung et al., 2017).

One of the significant factors that influence a newly graduated nurses' decision to resign from a job is feeling stress from taking care of patients without sufficient level of nursing experience and clinical judgment skills (Lau et al., 2015). This issue is related to the current nursing shortage problem and will continue into the future. Ineffective nursing care without productive clinical judgment is harmful to patients and increases nursing care errors, near misses, and adverse events (Van Graan \& Williams, 2017).

This study focused on senior nursing students who are preparing to be professional nurses. To become a professional nurse, senior nursing students are required to develop critical thinking skills; especially, clinical judgment. Clinical reasoning is an essential part of nursing care. In recent years, clinical judgment skills have been identified as a crucial competency and integral component of professional nursing practice. Clinical judgment is an essential part of patient care (Tanner, 2006). Therefore, to perform effective and productive nursing care to patients, nursing students need to improve this skill.

Clinical judgment is also grounded in the nurses' knowledge and experience and is a crucial element of productive and safe nursing practice. Lack of clinical judgment among senior nursing students is an added stress for them, 
particularly for those who deliver care primarily to critically ill patients. Furthermore, low clinical judgment skills that create higher personal stressors may impact the quality of nursing care and negatively affect patients' outcomes. In addition, clinical judgment is viewed as a problem-solving activity, starting with assessment and nursing diagnosis, and concluding in the evaluation of the effectiveness of interventions (Tanner, 2006; Lasater, 2007).

Clinical judgment linked to systematic problem solving involves an ability to consider and identify problems. It includes the ability to analyze problems and develop multiple solutions and choose the optimal solution (Tanner, 2006). Thus, this ability is required of senior nursing students who will become professional nurses. Senior nursing students' understanding of the concept of clinical judgment in nursing is crucial and essential; it is based on the relationship between the student's knowledge base, reasoning, and critical thinking skills. Clinical reasoning influences the process of how the senior nursing students improve effective nursing performance and ultimately decision making. How senior nursing students provide interpretations or conclusions regarding patients' needs, concerns, or health problems is an important key and relates to clinical judgment skill.

Clinical education is a crucial element in nursing education curriculum. Nursing students at all levels look forward to practicing and gaining nursing knowledge and experience in caring for patients. The performance of productive and effective nursing care is required of senior nursing students who are near graduation and becoming professional nurses.

There are various teaching tools and strategies that nursing educators use to assist nursing students in gaining knowledge and skill in nursing practice. Simulation is one excellent example of a teaching strategy that can encourage and guide nursing students to think critically and improve their clinical judgment (Jeffries, 2012). Simulation in nursing education has become increasingly prevalent for teaching students through a variety of clinical nursing skills. Additionally, high-fidelity simulation affords nursing students with the opportunity to gain more nursing experience in an environment that cannot harm patients. Moreover, simulation allows students to gain experience with complex patient situations that they might not get practicing in clinical settings. High-fidelity simulation can provide complex experiences for nursing students and guide them in recognizing potential life-threatening cues in a safe environment, which also develops their clinical judgment skills (Jeffries, 2012). Hyunsook et al. (2015) examined various levels of simulation experiences with groups of nursing students. The results showed that nursing students who participated with various simulations had significant increased level of thinking skills, especially clinical judgment and critical thinking. Senior nursing students need to be able to judge their decisions in patient care situations. Senior nursing students' development in clinical judgment skills through learning with high-fidelity simulation will be examined in this study.

The purpose of the research is to describe senior nursing students' experience in using high-fidelity simulation to evaluate the development of clinical judgment skills. For this purpose, answer to the following questions have been sought:

1) What is the relationship between senior nursing students' clinical judgment skills and learning with high-fidelity simulation?

2) What are the perceptions and experiences of senior nursing students on using high-fidelity simulation to support their clinical judgment skills?

3) To what extent and in what ways do the interviews with senior nursing students provide comprehensive understanding and development of clinical judgment skills from using high-fidelity simulation through integrative mixed-methods analysis?

\subsection{Hypotheses}

Null hypothesis (H0): There will be no difference in the means of senior nursing students' clinical judgment skills before and after participating in using high-fidelity simulation.

Alternative hypothesis (H1): There will be a difference in the means of senior nursing students' clinical judgment skills before and after participating in using high-fidelity simulation.

\section{Method}

\subsection{Identify Subsections}

A convergent, mixed-method design was used in this study that consists of a survey and semi-structured interviews. The advantage of the mixed-method design is that it allows some research questions to be addressed more comprehensively than by using quantitative or qualitative design alone. Particularly, a stronger study can result when using this design. This approach also allows the researcher to use data collection instruments for examining 
the research problem rather than only using limited types of data collection instruments such as quantitative or qualitative research (Creswell, 2011). The intent in using this design is to bring together the differing strengths and nonoverlapping weaknesses of both quantitative and qualitative methods. Each type of data, both qualitative and quantitative, can be collected and analyzed separately and independently, using techniques traditionally associated with each data type (Creswell, 2011).

\subsection{Participant Characteristics}

Full and part-time senior nursing students enrolled in the Bachelor of Science in Nursing (BSN) program at a selected School of Nursing are the target population of this study.

There are inclusion/exclusion criteria as follow:

The participants are senior nursing students who meet inclusion criteria as follows:

1) Participants are full and part-time pre-licensure students attending the Bachelor of Science in Nursing (BSN) program.

2) Participants are participating in clinical courses through the selected School of Nursing.

3) There is no exclusion criteria based on age, gender, or nationality.

4) Participants must be in good standing with the selected School of Nursing and nursing program.

\subsection{Sampling Procedures and Sample Size}

This study uses a convenience sampling method. A nonprobability sample is chosen because the study collected exploratory data from participants who were selected by nonrandom methods. The desired sampling size for the study is a minimum of 30 student nurses, however, sampling may be limited by course enrollment. Burns et al. (2013) indicated the ease of acquiring a convenience sample. In addition, convenience samples also allow researchers to obtain information in unexplored areas and these samples are accessible and usually require less time to acquire rather than other types of samples (Burns et al., 2013). Likewise, Lee-Jen et al. (2014) supported that the convenience sample is a type of nonprobability where members of the target population must meet certain practical criteria. The main object of convenience sampling is to collect information from participants who are easily accessible to the researcher. Additionally, the convenience sampling can be used in both qualitative and quantitative study including the mixed method study (Lee-Jen et al., 2014).

\subsection{Research Instrument}

Three instruments and a demographic survey were used for data collection for this study. The Lasater Clinical Judgment Rubric (LCJR), the Clark Simulation Evaluation Rubric (CSER), and semi-structured interview questions were used in this study. A researcher developed survey was used to collect student demographic data. LCJR is used for measuring senior nursing students' clinical judgment, the Lasater Clinical Judgment Rubric (LCJR) was used before and after participating with high-fidelity simulation. This rubric has four dimensions which consist of noticing, interpreting, responding, and reflecting during the clinical judgment process. Participants were evaluated on a total of 11 items: beginning ( 1 point), developing ( 2 points), accomplished ( 3 points), or exemplary (4 points). Therefore, the overall score is range from 11 to 44 on a clinical judgment scoring sheet. Participants completed self-reported sheets following a simulation activity. In addition the particpants completes a self-reflection through a semi-structured interview. The LCJR is used after obtaining permission from its author.

The CSER is the second instrument used for measuring senior nursing students' clinical judgment skills. The CSER is used to assess and evaluate nursing students' performance through practice with high-fidelity simulation. There are five main categories consisting of patient assessment/reassessment, history gathering, patient teaching, laboratory data and diagnostics, nursing interventions, clinical judgment, and communication. This instrument has a score range between 0 and 5 in each category.

The demographic data survey was developed by the researcher; it was used to ensure consistency and homogeneity within the group. The participants' demographic data allows the researcher to better understand certain background characteristics of each participant regarding participants' age, gender, race, ethnicity, level of education, academic year, and simulation experience.

\subsection{Mixed Methods Procedures and Data Collection}

In a convergent mixed-method design, quantitative and qualitative data are collected and analyzed during a similar timeframe as in the following diagram: 


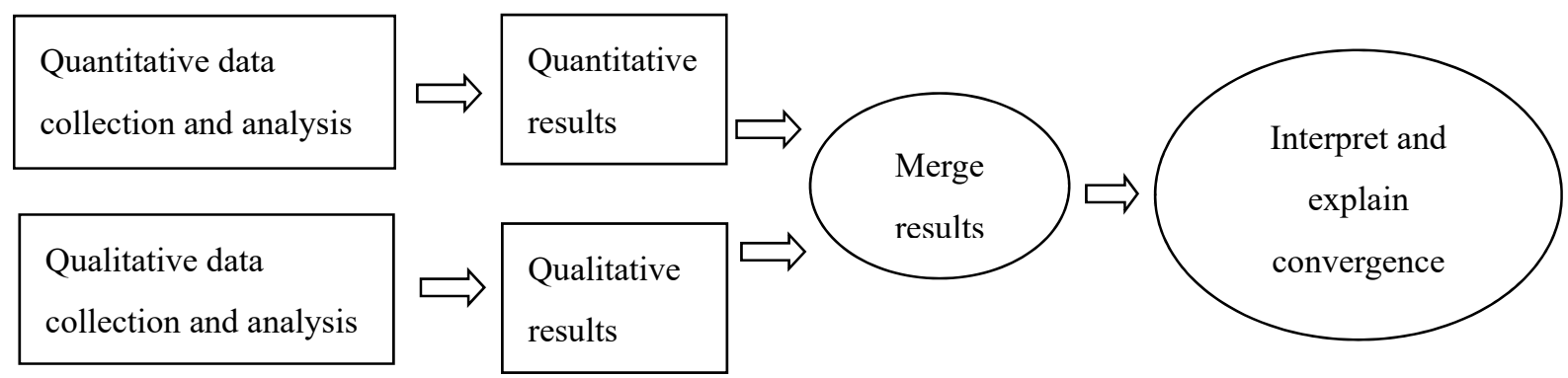

\subsection{Statistical Analysis}

The demographic data was analyzed using descriptive statistics to describe the sample using SPSS software. Regarding the LCJR and CSER, a $t$-test was used for a group comparison in terms of outcomes; especially paired t-test was used to compare means regarding research hypotheses. Statistical significance for this project is set at $p$ $=0.05$ level. Moreover, semi-structured interviews were interpreted manually and transcribed for coding regarding the number of participants. Regarding qualitative data analysis by using the semi-instructed interview section, there were three main steps of data analysis process that consist of the First Cycle Coding, Second Cycle, and analytical memos. The coding provided the researcher with an understanding of what is significant from the participants and also guide the researcher to preserve participants' meanings of senior nursing students' perceptions and experiences of using high fidelity simulation. For data integration, the quantitative and qualitative data were merged using a side-by-side comparison and jointly displaying both forms of data (Creswell, 2015). For this study, the quantitative results were reported first, followed by the qualitative results based on research questions.

\section{Results}

The main purpose of this mixed methods study was to describe senior nursing students' experiences in using high-fidelity simulation to evaluate the development of clinical judgment skills. The results are represented in the following three sections as quantitative, qualitative, and mixed method findings.

\subsection{Quantitative Results}

The purpose of the quantitative findings is to measure the relationship between senior nursing students' clinical judgment skills and learning with high-fidelity simulation by using a questionnaire. The quantitative research question was "What is the relationship between senior nursing students' clinical judgment skills and learning with high-fidelity simulation?"

Table 1. Frequencies and percentages for participants' demographic data variables $(\mathrm{N}=30)$

\begin{tabular}{llll}
\hline Variable & Category & $\mathrm{N}$ & $\%$ \\
\hline \multirow{3}{*}{ Age } & $18-24$ & 23 & 76.6 \\
& $25-30$ & 4 & 13.3 \\
& $31-34$ & 1 & 3.3 \\
\hline \multirow{2}{*}{ Gender } & $35-40$ & 2 & 6.7 \\
\hline \multirow{3}{*}{ Race/Ethnicity } & Male & 1 & 3.3 \\
& Female & 29 & 96.7 \\
\hline Level of education & Black or African American & 2 & 6.7 \\
& White & 23 & 76.7 \\
\hline Academic year & Asian & 2 & 6.7 \\
Simulation experience & Hispanic/Latino & 3 & 10 \\
\hline
\end{tabular}


All participants in the study were in their senior academic year and had prior simulation experience. The range of the participants' ages was 18 to 40 years with $76.7 \%$ participants between 18 to 24 years of age and $13.3 \%$ of the participants were 25 to 30 years old. Only $3.3 \%$ and $6.7 \%$ were 36 to 40 and over 40 years old, respectively. Moreover, the majority of participants were female (96.7\%), only one participant was a male. The race and ethnicity of participants in this study consist of Black or African American, White/Caucasian, Asian, and Hispanic or Latino. The majority of the participants were White/Caucasian (76.6\%), whereas Hispanic or Latino, African American, and Asian were minority groups; $10 \%, 6.7 \%$, and $6.7 \%$ respectively. Most of the participants had previously received bachelor's degrees as their highest level of education (93.3\%) and 6.7\% of participants had master's degrees.

Table 2. Paired sample t-test comparing before and after learning with HFS (senior nursing students on LCJR)

\begin{tabular}{lllllll}
\hline \multirow{2}{*}{ Dimension/Source } & \multicolumn{2}{l}{ Paired Differences } & & \\
\cline { 2 - 7 } & Mean & SD & Std. Error Mean & t value & df & $p$ \\
\hline Noticing & & & & & & \\
$\quad$ Focused observations & 1.10 & .61 & .11 & 9.92 & 29 & .000 \\
$\quad$ Recognizing deviations & 1.17 & .60 & .11 & 10.80 & 29 & .000 \\
$\quad$ Information seeking & 1.03 & .67 & .12 & 8.46 & 29 & .000 \\
\hline Interpreting & & & & & & \\
$\quad$ Prioritizing data & 1.07 & .64 & .12 & 9.13 & 29 & .000 \\
$\quad$ Making sense of data & .97 & .61 & .11 & 8.61 & 29 & .000 \\
\hline Responding & & & & & & \\
$\quad$ Calm confident manner & .93 & .64 & .12 & 8.00 & 29 & .000 \\
$\quad$ Clear communication & .93 & .58 & .11 & 8.76 & 29 & .000 \\
$\quad$ Well-planned intervention & 1.07 & .58 & .11 & 10.01 & 29 & .000 \\
$\quad$ Being skillful & 1.07 & .83 & .15 & 7.06 & 29 & .000 \\
\hline Reflecting & & & & & & \\
$\quad$ Evaluation/self-analysis & .50 & .57 & .10 & 4.79 & 29 & .000 \\
$\quad$ Commitment to improvement & .60 & .62 & .11 & 5.29 & 29 & .000 \\
\hline$p<.001$. & & & & &
\end{tabular}

Data were analyzed using paired t-test to evaluate the relationship between students' self-reported performance before and after a high-fidelity simulation scenario. An analysis of the data from the LCJR showed that the participants scored themselves higher in all dimensions after participating in a high-fidelity simulation than before the simulation occurred. Particularly, the relationship between before and after learning with high-fidelity simulation was a positive significant finding (Table 2.) at $p<0.000$, supporting the self-perceived improvement in the participants' clinical judgment skills following high-fidelity simulation.

Data analysis suggests (Table 2), student performance following high-fidelity simulation was positively associated with the development of student clinical judgment skills as measured by the LCJR assessment tool. Specially, results related to each dimension of LCJR are as follow (Table 2). Regarding the results, each item consist of focused observation (Mean $=1.10, \mathrm{SD}=.61, \mathrm{t}=9.92)$, recognizing deviations from expected patterns (Mean $=$ $1.17, \mathrm{SD}=.60, \mathrm{t}=10.80)$, information seeking (Mean $=1.03, \mathrm{SD}=.67, \mathrm{t}=8.46)$, prioritization (Mean $=1.07$, $\mathrm{SD}$ $=.64, \mathrm{t}=9.13)$, making sense of data $($ Mean $=.97, \mathrm{SD}=.61, \mathrm{t}=8.61)$, calm confident manner $($ Mean $=.93, \mathrm{SD}$ $=.64, \mathrm{t}=8.00$ ), clear communication (Mean $=.93, \mathrm{SD}=.58, \mathrm{t}=8.76$ ), well-planned intervention (Mean $=1.7, \mathrm{SD}$ $=.58, \mathrm{t}=10.01)$, being skillful (Mean $=1.07, \mathrm{SD}=.83, \mathrm{t}=7.06)$, evaluation/self-analysis (Mean $=.50, \mathrm{SD}=.57$, $\mathrm{t}=4.79$ ), and commitment to improvement (Mean $=.60, \mathrm{SD}=.62, \mathrm{t}=5.29)$. According to the data, senior nursing students who participated in high-fidelity simulation had an increase in their clinical judgment skills based on their self-perceived. The data showed the statistical significance in the development of clinical judgement skills of simulation participants. 
Table 3. Paired sample $t$-test comparing before and after learning with HFS of senior nursing students on CSER

\begin{tabular}{lllllll}
\hline \multirow{2}{*}{ Dimension/Source } & \multicolumn{2}{l}{ Paired Differences } & & \\
\cline { 2 - 7 } & Mean & SD & Std. Error Mean & t value & df & $p$ \\
\hline Patient assessment/reassessment & 1.27 & .52 & 1.46 & 13.32 & 29 & .000 \\
History gathering & 1.20 & .48 & 1.38 & 13.57 & 29 & .000 \\
Clinical judgment & 1.40 & .50 & 1.58 & 15.39 & 29 & .000 \\
Laboratory data and diagnostic & .93 & .58 & 1.15 & 8.76 & 29 & .000 \\
Nursing interventions & 1.33 & .47 & 1.51 & 15.23 & 29 & .000 \\
Communication & 1.00 & .37 & 1.14 & 14.75 & 29 & .000 \\
\hline$p<.001$. & & & & & &
\end{tabular}

As represented in Table, student performance following high-fidelity simulation was positively associated with the development of the student's clinical judgment skills as measured by the CSER assessment tool at $p<0.000$ (Table 8 ), there were five categories which consist of patient assessment/reassessment (Mean $=1.27, \mathrm{SD}=.52, \mathrm{t}=13.32$ ), history gathering (Mean $=1.20, \mathrm{SD}=.48, \mathrm{t}=13.57)$, clinical judgment $($ Mean $=1.40, \mathrm{SD}=.50, \mathrm{t}=15.39$ ), laboratory data and diagnostics (Mean $=.93, \mathrm{SD}=.58, \mathrm{t}=8.76$ ), nursing interventions (Mean $=1.33, \mathrm{SD}=.47, \mathrm{t}=$ 15.23), and communication (Mean $=1.00, \mathrm{SD}=.37, \mathrm{t}=14.75)$.

\subsection{Qualitative Results}

Semi-structured interviews were interpreted manually. Two main themes of senior nursing students who participated with high-fidelity simulation emerged from the data: senior nursing students' perceptions while participating with high-fidelity simulation and their experiences while participating with high-fidelity simulation. Both themes include subthemes that consist of the meaning and importance of using high-fidelity simulation and the purpose of high-fidelity simulation, learning progress and opportunity, prioritization, clinical judgment skill, and teamwork.

\subsubsection{Perceptions from Participating with High-Fidelity Simulation}

The interviews showed that senior nursing students' perceptions of participating with high-fidelity simulation related to the meaning and importance of using high-fidelity simulation and the purpose of high-fidelity simulation.

\section{The meaning and importance of using high-fidelity simulation}

High fidelity simulation is widely used in nursing education and has proven effective in optimizing clinical training. Most participants perceived that high-fidelity simulation provide participants with a non-threatening environment that supports them in developing their thinking, learning, and nursing skills. Particularly, the high-fidelity simulation that utilizes a computer-based mannequin. Most participants believed that the safe environment provided during participating with high-fidelity simulation is an important factor that can help and support their learning, especially the realistic situation. For example, participants said: "I think, working with mannequins, there's just going to be some technical difficulties that happen. But I felt like, it was good to step back, and we kind of analyzed everything we did. And that's the good thing about Sim, it is you get to see and talk about why you do, what you do. And then discussing it with everyone. And working all that out really helps you to remember it." Moreover, one participant mentioned that "I would say today's simulation was a good experience for us. Because, like I have never been in the emergency department before, I wouldn't know what to expect for a gunshot victim. And so that gave me a good taste of what to expect." The type of simulation had positive impact on the participants' learning that related to their academic year accordingly.

\section{Purpose of high-fidelity simulation}

Participants understood and perceived the purpose of HFS, it can guide and help them to perform nursing care collectively and effectively including helping them to improve their clinical judgement skills which is based on their thought process. Regarding this study, all of participants understood and perceived the purpose of using HFS, especially the case simulation scenario that the participants were using, it was a more complicated case and had many health conditions.

\subsubsection{Experiences from Participating with High-Fidelity Simulation}

This theme emerged from data that showed all participants had positive experiences from using HFS as a learning 
strategy. This theme included learning progress and opportunity, prioritization, clinical judgment skill, and teamwork.

\section{Learning progress and opportunity}

HFS helps senior nursing students to provide effective and efficient nursing care to their patients. Many self-reported improvements in confidence. All of them mentioned HFS can help them learn from their mistakes and guide them to develop their thinking skills which can progress their nursing care and performances.

\section{Prioritization}

Most participant have experiences from participating with HFS that helped them to learn to prioritize their nursing care. Particularly, prioritization begins with determining immediate threats to a patient's life as a part of the initial assessment. There were many participants who mentioned their prioritization during practicing with HFS. For example: "So, to me, it just meant, I guess, the intensity of that critical care environment, and how important it is to prioritize your steps of care, especially in these situations, because someone could be, you know, very close to death, or just in a crucial situation like this. And so, it's important that we prioritize, and we go in there with a group of people that understand what their role is." Similarly, one participant stated, "Like prioritizing, like patient care and different. Like assessments and just trying to figure out like, what is like the most important thing to do."

\section{Clinical judgment skills}

Practicing with HFS can encourage nursing students to develop their thinking skills, especially clinical judgment skills can be improved through practice. Most participants echoed this statement, "It definitely helps with like our clinical judgment and kind of you know, thinking outside of the box. And you know, not just getting in like the Monday, you know, like, everything we're used to, you know, it helps us broaden our knowledge skills." Similarly, one participant believed, "Yeah, absolutely. Anytime that you're given a situation that you may be placed in, it gives you that clinical judgment ability and also an opportunity to improve for when it actually happens. You are better prepared."

\section{Teamwork}

Experience from practicing with HFS developed the senior nursing students' ability to work effectively with other students as a team. There are many participants who mentioned working as a team.

\subsection{Mixed Method Results}

Table 4. The correlations of before and after learning with HFS (senior nursing students on LCJR)

\begin{tabular}{|c|c|c|c|c|}
\hline Dimension & Before participating $^{a}$ & After participating ${ }^{a}$ & Correlation & Sig. \\
\hline \multicolumn{5}{|l|}{ Noticing } \\
\hline Focused observations & $2.57(.82)$ & $3.67(.48)$ & .675 & .000 \\
\hline Recognizing deviations & $2.57(.77)$ & $3.73(.45)$ & .647 & .000 \\
\hline Information seeking & $2.78(.82)$ & $3.80(.41)$ & .581 & .001 \\
\hline \multicolumn{5}{|l|}{ Interpreting } \\
\hline Prioritizing data & $2.70(.80)$ & $3.77(.43)$ & .595 & .001 \\
\hline Making sense of data & $2.63(.81)$ & $3.60(.56)$ & .651 & .000 \\
\hline \multicolumn{5}{|l|}{ Responding } \\
\hline Calm confident manner & $2.63(.76)$ & $3.57(.57)$ & .574 & .001 \\
\hline Clear communication & $2.83(.75)$ & $3.77(.43)$ & .626 & .000 \\
\hline Well-planned intervention & $2.60(.67)$ & $3.67(.48)$ & .533 & .002 \\
\hline Being skillful & $2.47(.86)$ & $3.53(.57)$ & .388 & .034 \\
\hline \multicolumn{5}{|l|}{ Reflecting } \\
\hline Evaluation/self-analysis & $3.37(.67)$ & $3.86(.43)$ & .531 & .003 \\
\hline Commitment to improvement & $3.33(.71)$ & $3.93(.25)$ & .510 & .004 \\
\hline
\end{tabular}


Using two different methods for data collection, a quantitative survey, and qualitative semi-structured interviews, were done to provide a comprehensive understanding and development of senior nursing students' clinical judgment skills from using HFS. The quantitative and qualitative data were merged using a side-by-side comparison. The mixed methods research question was: "To what extent and in what ways do the interviews with senior nursing students provide comprehensive understanding and development of clinical judgment skills from using HFS through integrative mixed-methods analysis?"

In the quantitative strand, when looking at each dimension, the results showed after participating in a HFS mean scores were higher than before learning with HFS, significant findings (Table 4.). Especially, the highest mean score was found in recognizing deviations from expected patterns regarding comparing between before and after learning with HFS (Mean $=2.57, \mathrm{SD}=.77$ and Mean $=3.73, \mathrm{SD}=.45$ ). Senior nursing students can recognize the most obvious patterns and deviations in data and use these to continually assess. In addition, high mean scores were found in information seeking, prioritizing data, and clear communication regarding the mean score after participating in HFS (Mean $=3.80, \mathrm{SD}=.41$, Mean $=3.77, \mathrm{SD}=.43$, and Mean $=3.77, \mathrm{SD}=.43$, respectively). Senior nursing students focused on the important data and seek further relevant information to support planning interventions. Moreover, senior nursing students can communicate well.

These points are similar to the qualitative findings. All participants reported their perceptions and experiences from using HFS helped to develop and support their clinical judgment skills from the beginning through the end of the simulation, especially improving prioritizing data and working as a team with providing effective communication. Many participants mentioned similarly, "You just need to be able to prioritize all of the different things and how to prioritize, clinical judgment definitely helps." And, "It's important that we prioritize, and we go in there with a group of people that understand what their role is." Also, "I felt positive because the group that I worked with, worked really well with each other. good teamwork and everything." Finally, "Overall, I would have to say that communication with my team went really well for me."

According to the quantitative finding, there was a high mean score level of the clinical judgment dimension (Mean $=1.40, \mathrm{SD}=.50$ ).It indicated that participants could provide care while managing multiple contingencies and see the big picture of patient conditions well. The qualitative findings also indicate participants developed their clinical judgment skills through learning with HFS.

For example, one of the participants mentioned: "Yes, I think for sure. I think we were the first class that took the class clinical judgment. So, seeing those principles like put into play and actually being able to give our judgment and weigh the situation while we're doing it. I think it's been really helpful, especially in clinical now that we're doing leadership clinical, where we're expected to be a little bit more like taking on the role of our nurse. I think it's been really helpful from learning that through sim to be able to apply it in real life."

And "I think clinical judgment skill is incredibly important. I'm learning it from the beginning and being able to apply it". Moreover, all participants mentioned that high-fidelity simulation has a positive impact on their nursing and thinking skills. Therefore, the quantitative and qualitative data provided a comprehensive understanding and development of senior nursing students' clinical judgment skills from using HFS.

\section{Discussion}

A convenience sample of 30 senior nursing students were recruited to participate in this study using HFS. All participants in the study were in their senior academic year and had prior simulation experience. Participants who met the inclusion criteria and signed the informed consent and attended the selected school of nursing in the fall of 2020 were included in this study. Data was collected in two types: quantitative and qualitative data. The quantitative data was collected by two instruments, the Lasater Clinical Judgment Rubric (LCJR) and Clark Simulation Evaluation Rubric (CSER) questionnaires. Both instruments were used by students to self-evaluate their performance before and after a HFS scenario. The researcher collected self-evaluated questionnaires distributed through in-person and electronic mail from the participants. The qualitative data was collected by using semi-structured interviews, the interviews were conducted using the Zoom meeting program and/or interviews in-person with the researcher. The purpose of this interview is to establish a qualitative approach, that explored in-depth, the participants' experience through learning with HFS.

\subsection{Quantitative Findings}

The two instruments used to collect the quantitative data were the LCJR and CSER. Both instruments were used by nursing students to self-evaluate their performance before and after learning with HFS. The quantitative results showed the participants scored themselves higher in all dimensions after learning with HFS. Particularly, the relationship between before and after learning with HFS was a significant finding at as the analysis of the data from 
the LCJR. Also, the results supported the improvement in the participants' clinical judgment skills following HFS. As represented in Table 3, student performance following HFS was positively associated with the self-reported development of clinical judgment skills as measured by the CSER.

These results are consistent with those found by Craig et al. (2021), Amendoeira et al. (2020), Yang et al. (2019), Victor (2017), Bussard (2016), Chmil et al. (2015), and Aschcraft et al. (2013). Yang et al. (2019) found that simulation participation enhanced nursing students' clinical judgment skills and the members of the experimental group performed better on their nursing care and responding on patient safety. Research by Ashcroft et al. (2013) noted that simulation participation helped nursing students in the development of clinical judgement when dealing with patient problems or issues within a simulated setting. Amendoeira et al. (2020) found that nursing students improved their clinical judgment skills; especially, the skills to interpret patient data. All of these studies support the use of HFS as an important teaching strategy for improving nursing students' clinical judgment skills.

Research by Victor (2017) supported that participating in simulation had a significant increase in clinical judgment at both the beginning and the end of the nursing program. Particularly, simulation can improve nursing students' outcomes by fostering the development of clinical judgment (Victor, 2017). Research by Bussard (2016) supported that nursing students developed clinical judgement skills after participating with HFS. Especially, during debriefing sessions that had immediately followed simulation scenarios as a method for nursing students to connect theory to practice (Bussard, 2016).

Research by Chmil et al. (2015) also supported that learning with HFS had positive effects on nursing students' clinical judgment development. There was a significant positive relationship between the development of clinical judgment and simulation performance (Chmil et al., 2015). Simulation was also found to be an effective strategy to increase nursing student clinical competence in safe medication administration practices (Craig, et al., 2021). Craig et al. (2021) reported that participating with HFS while participating safe medication administration can improve students' knowledge and competency in safe medication management and improve nursing students' thinking skills.

\subsection{Qualitative Findings}

Analysis of the qualitative data led to the emergence of two main themes: Students'perceptions and experiences in learning with HFS. Both themes include subthemes relating to the meaning and importance of using high-fidelity simulation and the purpose of HFS, learning progress and opportunity, prioritization, clinical judgment skill, and teamwork.

\subsubsection{Students' Perceptions}

\section{- The meaning and importance of using HFS}

All participants acknowledged that HFS provide participants with a non-threatening environment that supports them in developing their thinking, learning, and nursing skills. Participants believed that the safe environment provided during participating with HFS is an important factor that helps and supports their learning because it provides a realistic situation. Similarly, research by Bland et al. (2011), completed a concept analysis of simulation as a learning strategy in the education of undergraduate nursing students. The findings identified HFS as realistic computer simulation that imitates and represents the replication of a patient's conditions. It is an effective learning strategy that can help nursing students improve their nursing skills. In addition, simulation offers a safe non-threatening environment that can create opportunities for nursing students to develop cognitive, psychomotor, and affective competencies away from the patient's bedside (Bland et al., 2011). A similar result occurred in Au et al. (2016), studied the perceptions of HFS as a clinical replacement in 80 first year nursing students' using an open-ended questionnaire. During the study, students participated in two HFS. The findings showed that nursing students have effective preparation for emergency situations while learning with HFS. The HFS provided nursing students with a realistic hospital environment (Au et al., 2016).

\section{- Purpose of HFS}

In addition, some participants stated that the purpose of using HFS guided them to recognize and learn from their mistakes and to improve their nursing skills when they become professional nurses. HFS helps prepare nursing students to provide effective and efficient nursing care to their patients. Many participants in this study reported improvements in self-confidence. All of the students mentioned HFS helped them learn from their mistakes and guided them to develop their thinking skills which can progress their nursing care and performances. This is consistent with the results of the study conducted by Kimhi et al. (2016). Kimhi et al. (2016) examined the impact of simulation and clinical experience on self-efficacy in 70 nursing students and supported that HFS improved nursing students' ability to apply the nursing process, organize nursing care, and perform technical skills, in 
addition to self-confidence.

\subsubsection{Experiences in Learning with HFS}

\section{- Learning progress and opportunity}

Many participants in this study self-reported improvements in confidence regarding their ability to provide effective and efficient nursing care. All of them mentioned HFS helped them learn from their mistakes and guide them in the development of their thinking skills, especially improving their nursing care and performances. In addition, students described debriefing as an opportunity to further enhance learning and participants' thinking skills. Moreover, the feedback from the facilitator had a positive impact on their learning, specifically the way the facilitator encourages them to think back to what they did through the simulation scenario.

Research by Lestander et al. (2016) supported that HFS provides a safe and realistic environment that can be used in nursing education. Especially, when the debriefing that follows a simulation experience provides opportunities for nursing students to analyze and reflect upon their decisions, actions, and outcomes (Lestander et al., 2016). Research by Woosuck et al. (2020) suggests that simulation provides nursing students with the opportunity to improve caring skills and clinical competence. In addition, simulation allows nursing students to increase their learning and to develop the roles and responsibilities that are part of the nursing profession. (Woosuck et al., 2020).

\section{- Prioritization}

Findings from this study showed most participants had learned prioritization skills by participating in HFS. Prioritization begins with determining immediate threats to a patient's life as a part of the initial assessment. There were many participants who mentioned the use of prioritization skills during simulation. Suk et al. (2015) studied the first experiences of HFSs training in 33 nursing students. The findings of this study identified that nursing students improved their learning abilities while participating with HFS, the majority of participants showed an increased ability identifying changes in the patients' condition, decision making, and debriefing in effectiveness of simulation. Moreover, nursing students learned to prioritize and determine immediate nursing care as part of the initial assessment (Suk et al., 2015). Similarly, research by Kaplan and Ura (2010) studied the use of multiple patient simulators to enhance prioritizing and delegating skills for senior nursing students. The findings of this study suggested that the simulation developed and increased nursing students' prioritizing and delegating skills and improved their knowledge and confidence while providing nursing care (Kaplan \& Ura, 2010).

\section{- Clinical judgment skills}

Regarding qualitative findings, learning with HFS encouraged nursing students to develop their clinical judgment skills especially improved through practice. A similar result was found in a study by Tosterud et al. (2013). Tosterud et al. (2013) studied nursing students' perceptions of high and low fidelity simulation, when simulation was used as a learning method. Eighty-six baccalaureate nursing students were randomly divided into groups participating in high and low-fidelity simulation. The results showed that students in HFS groups that imitated specialty units had a more positive experience and reported improved competency. Such experiences also facilitated their transition from student to clinical professional. Specifically, the participants of HFS felt their skills in the areas of clinical judgment, decision-making, problem-solving, prioritizing, awareness of personal feelings, and participation in teamwork are enhanced by practicing with high-fidelity simulation (Tosterud et al., 2013).

\section{- Teamwork}

Experience from learning with HFS developed the senior nursing students' ability to work effectively with other students as a team. There were many participants who mentioned working as a team. In any kind of nursing care situation, working as a team is an important action that nurses need to be able to clearly and effectively communicate with other healthcare professionals to solve the patients' illness problems and conditions. Particularly, collaboration within a group can help solve difficult problems. Similar results were reported by Foster et al. (2019). This study used simulation to develop teamwork skills in prelicensure nursing students. The findings of this study indicate that nursing students learned how to communicate with members of a healthcare team; for example, when to call a provider and speak up without fear of retribution. Moreover, nursing students were able to improve their problem-solving skills using and communicating skills using team approach (Foster et al., 2019). Additional research by Woosuck et al. (2020) identified that participating with HFS enhanced nursing students' collaboration and confidence in communication skills.

\subsection{Mixed Methods Findings}

The convergence of both quantitative and qualitative findings allowed the researcher to understand senior nursing students' experiences in using HFS to evaluate their development of clinical judgment skills. Regarding the 
research question and hypothesis, senior nursing students' clinical judgment improved by learning with high-fidelity simulation. Also, there was a difference in the means of senior nursing students' clinical judgment skills before and after learning with HFS supporting the hypothesis.

The highest mean score was found in "participants ability to recognize deviations from expected patterns of nursing care" in both the before and after learning with HFS. Senior nursing students recognized most obvious patterns and deviations in data and used these to continually assess.

In addition, higher mean scores were found in information seeking, prioritizing data, and clear communication regarding the mean score after learning with HFS. Senior nursing students were able to focus on the important data and sought additional relevant information to support planning interventions. Moreover, senior nursing students communicated well when providing their self-evaluation when learning with HFS.

All participants of the current study reported they believed that their experiences from using HFS developed and supported their clinical judgment skills from the beginning through the end of the simulation, especially improving prioritizing data and working as a team with providing effective communication. This is consistent with the results from other studies. Page-Cutrara and Turk (2017) reported that nursing students who participated with the simulation were able to improve their prioritization skills. In addition, nursing students were able to identify the appropriate nursing care plans for simulated patient care with guidance from a simulation facilitator (Page-Cutrara \& Turk, 2017).

A high mean score in the clinical judgment dimension indicated that participants could see the big picture regarding a patient's condition and provide care while managing multiple contingencies. The qualitative finding suggest that participants developed their clinical judgment skills through HFS and could answer the research questions and hypotheses. According to Lasater (2007), for the experienced nurse encountering a familiar situation, the nurse is able to respond intuitively based on immediate clinical grasp and knowing what to do. HFS supports Lasater's premise that clinical judgment is developed through practice, learning, experience, knowledge, and continuous critical analysis (Lasater, 2007).

\subsection{Limitations}

This study was administered in person by the researcher using convenience sampling with 30 senior nursing students who met the inclusion criteria. The number of participants was adequate for data analysis, but it was small sample size depend on the inclusion criteria; especially, relating to senior nursing students who attended the Bachelor of Science in Nursing (BSN) program. A larger sample size could have generated more representative results.

\section{Competing Interests Statement}

The authors declare that there are no competing or potential conflicts of interest.

\section{References}

Amendoeira, J. J. P., Carvalho, E. C., Cruz Diná, A. L., Lasater, K., Morais, S. C. R. V., \& Nunes, J. G. P. (2020). Clinical judgment and diagnostic reasoning of nursing students in clinical simulation. Revista Brasileira De Enfermagem, 73(6). https://doi.org/10.1590/0034-7167-2018-0878

American Nurses Association (2016). Nurse staffing crisis. https://www.nursingworld.org/practice-policy/nurse-staffing/nurse-staffing-crisis/

Ashcraft, A. S., Opton, L., Bridges, R. A., Caballero, S., Veesart, A., \& Weaver, C. (2013). Simulation evaluation using a modified Lasater clinical judgment rubric. Nursing Education Perspectives, 34(2), 122-6.

Au, M. L., Lo, M. S., Cheong, W., Wang, S. C., \& Van, I. K. (2016). Nursing students' perception of high-fidelity simulation activity instead of clinical placement: A qualitative study. Nurse Education Today, 39, 16-21. https://doi.org/10.1016/j.nedt.2016.01.015

Bland, A. J., Topping, A., \& Wood, B. (2011). A concept analysis of simulation as a learning strategy in the education of undergraduate nursing students. Nurse Education Today, 31(7), 664-670. https://doi-org.ezproxy.okcu.edu/10.1016/j.nedt.2010.10.013

Burns, N., Grove, S. K., \& Gray. J. R. (2013). The practice of nursing research: Appraisal, synthesis and generation of evidence (7th ed.). St. Louis: Saunders Elsevier. ISBN-13: 978-0323377584

Bussard, M. E. (2016). Self-reflection of video-recorded high-fidelity simulations and development of clinical judgment. The Journal of Nursing Education, 55(9), 522-7. https://doi.org/10.3928/01484834-20160816-06 
Chmil, J. V., Turk, M., Adamson, K., \& Larew, C. (2015). Effects of an experiential learning simulation design on clinical nursing judgment development. Nurse Educator, 40(5), 228-32. https://doi.org/10.1097/NNE.0000000000000159

Craig, S. J., Kastello, J. C., Cieslowski, B. J., \& Rovnyak, V. (2021). Simulation strategies to increase nursing student clinical competence in safe medication administration practices: A quasi-experimental study. Nurse Education Today, 96, 104605-104605. https://doi.org/10.1016/j.nedt.2020.104605

Creswell, J. W. (2011). Designing and conducting mixed methods research. Los Angeles: SAGE Publication.

Foster, M., Gilbert, M., Hanson, D., Whitcomb, K., \& Graham, C. (2019). Use of simulation to develop teamwork skills in prelicensure nursing students: an integrative review. Nurse Educator, 44(5), 11. https://doi.org/10.1097/NNE.0000000000000616

Hyunsook Shin, Hyunhee Ma, Jiyoung Park, Eun Sun Ji, \& Dong Hee Kim. (2015). The effect of simulation courseware on critical thinking in undergraduate nursing students: Multi-site pre-post study. Nurse Education Today, 35(4), 537-542.

Jeffries, P. (2012). Simulation nursing education: From conceptualization to evaluation ( $2^{\text {nd }}$ ed.). New York, NY: Laerdal Medical Corporation.

Jung, D., Lee, S. H., Kang, S. J., \& Kim, J. (2017). Development and evaluation of a clinical simulation for new graduate nurses: A multi-site pilot study. Nurse Education Today, 49, 84-89. https://doi.org/10.1016/j.nedt.2016.11.010

Kaplan, B., \& Ura, D. (2010). Use of multiple patient simulators to enhance prioritizing and delegating skills for senior nursing students. Journal of Nursing Education, 49(7), 371-377. https://doi-org.ezproxy.okcu.edu/10.3928/01484834-20100331-07

Kimhi, E., Reishtein, J. L., Cohen, M., Friger, M., Hurvitz, N., \& Avraham, R. (2016). Impact of simulation and clinical experience on self-efficacy in nursing students: Intervention study. Nurse Educator, 41(1), E1-E4. https://doi-org.ezproxy.okcu.edu/10.1097/NNE.0000000000000194

Lasater, K. (2007). Clinical judgment development: Using simulation to create an assessment rubric. Journal of Nursing Education, 46(11), 496-503.

Lau, R., Willetts, G., Hood, K., \& Cross, W. (2015). Development of self-efficacy of newly graduated registered nurses in an aged care program. Australasian Journal on Ageing, 34(4), 224-228. https://doi.org/10.1111/ajag.12156

Lee-Jen Wu Suen, Hui-Man Huang, \& Hao-Hsien Lee. (2014). A comparison of convenience sampling and purposive sampling. Journal of Nursing, 61(3), 105-111. https://doi.org/10.6224/JN.61.3.105

Lestander, Ö., Lehto, N., \& Engström, ̊̊. (2016). Nursing students' perceptions of learning after high fidelity simulation: Effects of a three-step post-simulation reflection model. Nurse Education Today, 40, 219-224. https://doi-org.ezproxy.okcu.edu/10.1016/j.nedt.2016.03.011

Page-Cutrara, K., \& Turk, M. (2017). Impact of prebriefing on competency performance, clinical judgment, and experience in simulation: An experimental study. Nurse Education Today, 48, 78-83. https://doi-org.ezproxy.okcu.edu/10.1016/j.nedt.2016.09.012

NSI Nursing Solution. (2021). 2021 NSI National Health Care retention and RN staffing report. https://www.nsinursingsolutions.com/Documents/Library/NSI_National_Health_Care_Retention_Report.pd $\mathrm{f}$

Suk, J. Lee., Sang, S. K., \& Young-Mi, Park. (2015). First experiences of high-fidelity simulation training in junior nursing students in Korea. Japan Journal of Nursing Science, 12(3), 222-231. https://doi-org.ezproxy.okcu.edu/10.1111/jjns.12062

Sweeney, N. Lu., Rollins, M. C., Gantt, L., Swanson, M., \& Ravitz, J. (2020). Development and Reliability Testing of the Sweeney-Clark Simulation Evaluation Rubric C. Clinical Simulation in Nursing, 41, 22-32

Tanner, C. A. (2006). Thinking like a nurse: A research-based model of clinical judgment in nursing. Journal of Nursing Education, 45(6), 204-211.

Tosterud, R., Hedelin, B., \& Hall-Lord, M. L. (2013). Nursing students' perceptions of high- and low-fidelity simulation used as learning methods. Nurse Education in Practice, 13(4), 262-270. https://doi.org/10.1016/j.nepr.2013.02.002 
Van Graan, A. C., \& Williams, M. J. S. (2017). A conceptual framework to facilitate clinical judgement in nursing: A methodological perspective. Health SA Gesondheid, 22, 275-290. https://doi-org.ezproxy.okcu.edu/10.1016/j.hsag.2017.01.004

Victor, J. (2017). Improving clinical nursing judgment in prelicensure students. The Journal of Nursing Education, 56(12), 733-736. https://doi.org/10.3928/01484834-20171120-05

Woosuck, L., Miran, K., Yun, K., Yu-Jin, L., So, M. K., Janghoon, L., Soo-Jin, H., Jihye, Y., \& Young-Suk, P. (2020). Nursing and medical students' perceptions of an interprofessional simulation-based education: A qualitative descriptive study. Korean Journal of Medical Education, 32(4), 317-327. https://doi.org/10.3946/kjme.2020.179

Yang, F., Wang, Y., Yang, C., Zhou, M. H., Shu, J., Fu, B., \& Hu, H. (2019). Improving clinical judgment by simulation: A randomized trial and validation of the Lasater clinical judgment rubric in Chinese. $B M C$ Medical Education, 19(1), 20-20. https://doi.org/10.1186/s12909-019-1454-9

\section{Copyrights}

Copyright for this article is retained by the author(s), with first publication rights granted to the journal.

This is an open-access article distributed under the terms and conditions of the Creative Commons Attribution license (http://creativecommons.org/licenses/by/4.0/). 